\title{
Search for Standard Model Higgs boson decaying into 4 leptons with CMS detector
}

\author{
M. Dalchenko* \\ Laboratoire Leprince-Ringuet, Palaiseau, France \\ E-mail: mykhailo.dalchenko@cern.ch
}

\section{Kovač}

Technical University of Split, Croatia and Laboratoire Leprince-Ringuet, Palaiseau, France

E-mail: marko.kovac@cern.ch

\section{R. Pleština}

Technical University of Split, Croatia and Laboratoire Leprince-Ringuet, Palaiseau, France

E-mail: roko.plestina@cern.ch

\begin{abstract}
A search for the Higgs boson in the $H \rightarrow Z Z$ four-lepton decay channel, with each $Z$ boson decaying to an electron, or a muon pair, is reported. The search covers Higgs boson mass hypotheses in the range $110<m_{H}<600 \mathrm{GeV}$. The analysis uses pp collision data recorded by the CMS detector at the LHC, corresponding to integrated luminosities of $5.05 \mathrm{fb}^{-1}$ at $\sqrt{s}=7 \mathrm{TeV}$ and $5.26 \mathrm{fb}^{-1}$ at $\sqrt{s}=8 \mathrm{TeV}$. The four-lepton invariant-mass distributions $m_{4 \ell}$ is found to be consistent with the standard model expectations for background $\mathrm{ZZ}$ production for invariant masses above 180 $\mathrm{GeV}$. Upper limits at $95 \%$ confidence level exclude the standard model Higgs boson in the range 131-162 and 172-525 GeV, while the expected exclusion range is $121-570 \mathrm{GeV}$. An excess of events is observed in the low $m_{4 \ell}$ mass range, making the observed limits weaker than expected in the absence of a signal. These events cluster around a mass $m_{4 \ell} \simeq 125.5 \mathrm{GeV}$, giving rise to a local excess with respect to the background expectation, with a significance of $3.2 \sigma$. This result constitutes evidence for a new massive state.
\end{abstract}

36th International Conference on High Energy Physics,

July 4-11, 2012

Melbourne, Australia

\footnotetext{
*Speaker.

${ }^{\dagger}$ Speaker.

${ }^{\dagger}$ Speaker.
} 


\section{Introduction}

The standard model (SM) of electroweak interactions [1, 2, 3] relies on the existence of the Higgs boson $(H)$, a scalar particle of mass $m_{H}$ associated with the field responsible for the spontaneous electroweak symmetry breaking $[4,5,6,7,8,9]$. The value of $m_{H}$ is not fixed by the theory, and the existence of the scalar boson has not yet been established experimentally. The production of a Higgs boson, followed by its decay $H \rightarrow Z Z$, is expected to be one of the main discovery channels at the proton-proton (pp) Large Hadron Collider (LHC) [10] for a wide range of $m_{H}$ values.

Direct searches for the SM Higgs bosons have been performed by the ATLAS and CMS experiments using each about $5 \mathrm{fb}^{-1}$ of $p p$ data from the LHC collected in 2010 and 2011 at $\sqrt{s}=7 \mathrm{TeV}$. They include searches in the $H \rightarrow Z Z \rightarrow 4 \ell$ channel $(\ell=e, \mu)$ by ATLAS [11] and CMS [12]. The results from CMS exclude the SM Higgs boson in the mass range 127-600 GeVat 95\% confidence level (CL) [13].. ATLAS excludes 111.4-116.6 GeV, 119.4-122.1 GeV, and 129.2- 541 $\mathrm{GeV}$ at $95 \% \mathrm{CL}[14,15]$. Direct searches for the SM Higgs boson at the LEP $e^{+} e^{-}$collider and the Tevatron $p \bar{p}$ collider have led, respectively, to a lower-mass bound of $m_{H}>114.4 \mathrm{GeV}$ [16], and to an exclusion in the range $162-166 \mathrm{GeV}$ [17], at 95\% CL. Indirect constraints from precision measurements favour the mass range $m_{H}<158 \mathrm{GeV}[18,19]$ at 95\% CL.

In this poster, a search in the four-lepton decay channels $H \rightarrow 4 \ell$, with $\ell=e$ or $\mu$, is presented. The analysis is designed for a Higgs boson in the mass range $110<m_{H}<600 \mathrm{GeV}$. It re-uses the data collected at $\sqrt{s}=7 \mathrm{TeV}$ by CMS in 2011, corresponding to an integrated luminosity of $5.05 \mathrm{fb}^{-1}$, combined with new data collected in 2012 at $\sqrt{s}=8 \mathrm{TeV}$, corresponding to an additional $5.26 \mathrm{fb}^{-1}$.

\section{Experimental methods and Datasets}

The full description of the CMS detector can be found in Ref. [20]. A complete reconstruction of the individual particles emerging from each collision event is obtained via a particle-flow (PF) technique. This uses the information from all CMS sub-detectors to identify and reconstruct individual particles in the collision event [21, 22], with particles classified into mutually exclusive categories: charged hadrons, neutral hadrons, photons, muons, and electrons. For electrons, the basic collection of reconstructed candidates is first obtained separately within the geometrical acceptance, $\left|\eta^{e}\right|<2.5$, and for $p_{T}^{e}>7 \mathrm{GeV}$. The reconstruction combines the information from clusters of energy deposits in the ECAL and the trajectory in the inner tracker [23, 24]. Muons are reconstructed within $\left|\eta^{\mu}\right|<2.4$ and for $p_{T}^{\mu}>5 \mathrm{GeV}$ combining the information from both the silicon tracker and the muon spectrometer [25].

The isolation of individual $e$ or $\mu$ leptons is measured relative to their transverse momentum $p_{T}^{\ell}$, by summing transverse momentum(energy) over charged(neutral) particles in a cone $\Delta R=$ $\sqrt{\left(\eta^{\ell}-\eta^{i}\right)^{2}+\left(\phi^{\ell}-\phi^{i}\right)^{2}}<0.4$ around the lepton direction at the interaction vertex:

$$
R_{\mathrm{Iso}}^{\ell} \equiv\left(\sum p_{T}^{\text {charged }}+\operatorname{MAX}\left[0, \sum E_{T}^{\text {neutral }}+\sum E_{T}^{\gamma}-\rho \times A_{\text {eff }},\right]\right) / p_{T}^{\ell} .
$$

The term $\rho \times A_{\text {eff }}$ subtracts an estimate obtained using a "jet area" technique [26] of the transverse energy from neutrals in the isolation cone coming from pileup of additional pp collisions. The electrons or muons are considered isolated if $R_{\mathrm{Iso}}^{\ell}<0.4$. 
To ensure that electrons or muons pairs from $\mathrm{Z}$ decays originate from the primary vertex, a requirement on the significance of the impact parameter to the event vertex, $\mathrm{SIP}_{3 \mathrm{D}}$, is used, satisfying $\left|\mathrm{SIP}_{3 \mathrm{D}}=\frac{\mathrm{IP}}{\sigma_{\mathrm{IP}}}\right|<4$ for each lepton. The IP is the lepton impact parameter in three dimensions at the point of closest approach with respect to the primary interaction vertex, and $\sigma_{\mathrm{IP}}$ the associated uncertainty.

The efficiencies for the product of reconstruction, identification, and isolation of primary $e$ or $\mu$ leptons are measured in data, using a tag-and-probe technique [27] based on an inclusive sample of $\mathrm{Z}$ events.

Photons reconstructed within $\left|\eta^{\gamma}\right|<2.4$ are possible FSR candidates. To be accepted as FSR, a reconstructed photon must either have $p_{\mathrm{T}}^{\gamma}>2 \mathrm{GeV}$ and be found within a conical distance $\Delta R<$ 0.07 from a selected lepton candidate, or have $p_{\mathrm{T}}^{\gamma}>4 \mathrm{GeV}$ and be found isolated $\left(R_{\mathrm{Iso}}^{\gamma}<1\right)$ with $0.07<\Delta R<0.5$ around a selected lepton candidate.

Collision events are selected by the trigger system that requires the presence of a pair of electrons or a pair of muons. A cross-trigger requiring an electron and a muon is also used for the 2012 data. The requirements on the transverse energy (transverse momenta) for the first and second lepton are 17 and $8 \mathrm{GeV}$ respectively.

Monte Carlo (MC) samples for the SM Higgs boson signal and for background processes are used to optimize the event selection and to evaluate the acceptance and systematic uncertainties.

\section{Background control and Systematics}

We rely on MC simulation to evaluate the local density $\left(\Delta N / \Delta m_{4 \ell}\right)$ of events expected as a function of the mass $m_{4 \ell}$ from the ZZ background. The uncertainties for the QCD and PDF scales for each final state are on average $8 \%$.

To estimate the reducible $(Z b \bar{b}, t \bar{t})$ and instrumental $(Z+$ light jets, $W Z+$ jets) backgrounds, a $Z_{1}+X$ background control region, well separated from the signal region, is defined.

Two different approaches are used for measuring the rates.Both are based on relaxed isolation and identification criteria for two additional reconstructed lepton objects and proper extrapolation of rates to the signal region. Comparable background counts in the signal region are found within uncertainties from both methods. An envelope comprising these results is used as the final estimate.

Systematic uncertainties are evaluated from data for trigger (1.5\%), and combined lepton reconstruction, identification and isolation efficiencies (varying from $1.2 \%$ in the $4 \mu$ channel at high masses to about $11 \%$ in $4 e$ channel at low masses). The uncertainty associated with $\tau_{h}$ identification and isolation is $6 \%$. Uncertainties on $\tau_{h}$ energy scale (3\%) contribute to variation in the shape of the mass spectrum. Systematic uncertainties on energy-momentum calibration $(0.4 \%$ for muons and $0.2 \%$ for electrons), and energy resolution are accounted for by their effects on the reconstructed mass distributions. The effect of the energy resolution uncertainties is taken into account by introducing a $20 \%$ uncertainty on the simulated width of the signal mass peak. To validate the level of accuracy with which the absolute mass scale and resolution are known, we use $Z \rightarrow \ell \ell$ and $J / \psi \rightarrow \ell \ell$ events. Additional systematic uncertainties arise from limited statistical precision in the reducible background control regions. All reducible and instrumental background sources are derived from control regions, and the comparison of data with the background expectation in the 
signal region is independent of the uncertainty on the LHC integrated luminosity of the data sample. This uncertainty (4.4\%) [28] enters the evaluation of the ZZ background and in the calculation of the cross section limit through the normalisation of the signal. Systematic uncertainties on the Higgs boson cross section (17-20\%) and branching fraction (2\%) are taken from Ref. [29].

\section{Event selection and Kinematics}

The signal candidates in the $4 \ell$ analysis are selected using well identified and isolated primary leptons. The lepton isolation requirements suppress the $\mathrm{Z}+\mathrm{jet}, \mathrm{Z} b \bar{b}$ and $t \bar{t}$ backgrounds. The requirement on the significance of the impact parameter to the event vertex $\left|\operatorname{SIP}_{3 \mathrm{D}}\right|<4$ further suppresses the $\mathrm{Z} b \bar{b}$ and $t \bar{t}$ backgrounds. When building the $\mathrm{Z}$ candidates, only the FSR photons associated with the closest lepton and which make the "dressed" lepton-pair mass closer to the nominal $\mathrm{Z}$ mass are kept, with a maximum mass $m_{\ell \ell \gamma}<100 \mathrm{GeV}$. We require a $\mathrm{Z}$ candidate formed with a pair of leptons of the same flavour and opposite charge $\left(\ell^{+} \ell^{-}\right)$. The pair with an invariant mass closest to the nominal $\mathrm{Z}$ mass is denoted $m_{Z_{1}}$ and retained if it satisfies $40<m_{Z_{1}}<120 \mathrm{GeV}$. We then consider all remaining leptons and require a second pair of $\ell^{+} \ell^{-}$, with mass denoted $m_{Z_{2}}$, to satisfy $12<m_{Z_{2}}<120 \mathrm{GeV}$. The $12 \mathrm{GeV}$ cut provides an optimal sensitivity for a Higgs boson mass hypothesis in the range $110<m_{H}<160 \mathrm{GeV}$. Among the four selected leptons forming $Z_{1}$ and the $Z_{2}$, at least one should have $p_{T}>20 \mathrm{GeV}$ and another one have $p_{T}>10 \mathrm{GeV}$ to ensure that the selected events have leptons on the high-efficiency plateau for the trigger. To further protect against leptons originating from hadron decays in jet fragmentation or from the decay of low-mass hadronic resonances, we require that any opposite-charge pair of leptons chosen among the four selected leptons (irrespective of flavour) satisfy $m_{\ell \ell^{\prime}}>4 \mathrm{GeV}$. The phase space for the search of the SM Higgs boson is defined by restricting the mass range to $m_{4 \ell}>100 \mathrm{GeV}$. A higher minimal threshold on $m_{Z_{1}}$ and $m_{Z_{2}}$ could be used for higher $m_{H}$ values but only with marginal improvement of the sensitivity.

The event yields are found to be in good agreement with the MC background expectation at each step of event selection.

Kinematics of the Higgs or exotic boson decay to $Z Z$ final state has been extensively studied in the literature [30, 31, 32, 33, 32, 34, 35, 36, 37, 38, 39, 40, 41]. Since the Higgs boson is spinless, the angular distribution of its decay products is independent of the production mechanism. Five angles $\left(\theta^{*}, \Phi_{1}, \theta_{1}, \theta_{2}, \Phi\right)$ defined in Ref. [39] and the invariant masses of the lepton pairs, $m_{Z_{1}}$ and $m_{Z_{2}}$, fully describe the kinematics of the $H \rightarrow Z Z \rightarrow 4 \ell$ process at a given mass of the four-lepton system in their rest frame. These observables provide significant discriminating power between signal and background.

We use a matrix element likelihood analysis (MELA) and construct a kinematic discriminant $(\mathrm{KD})$ based on the probability ratio of the signal and background hypotheses, as described in Ref. [39]. The likelihood ratio is defined for each value of $m_{4 \ell}$. The signal and $q \bar{q} \rightarrow Z Z$ background analytical parametrisations are taken from Refs. [39] and [41], respectively, and include the phase-space and $Z$ propagator terms. When $m_{4 \ell}$ is above the $2 m_{Z}$ threshold, the two $Z$ bosons are on-shell and no separation is provided by $m_{Z_{1}}$ and $m_{Z_{2}}$, therefore leaving only the five angles in the parametrisation. When $m_{4 \ell}$ is below the $2 m_{Z}$ threshold, background has significant contribution 
from the $q \bar{q} \rightarrow Z Z^{(*)} / Z \gamma^{(*)}$ processes and instead of analytical parametrisation it is tabulated in a correlated template distribution using POWHEG simulation at generator level.

\section{Results}

The distribution of the four-lepton reconstructed mass for the sum of the $4 e, 4 \mu$, and $2 e 2 \mu$ channels, and the distribution of the MELA KD versus the four-lepton reconstructed mass $m_{4 \ell}$ are shown in Fig. 1 in the low mass range. The background shapes are taken from MC simulation
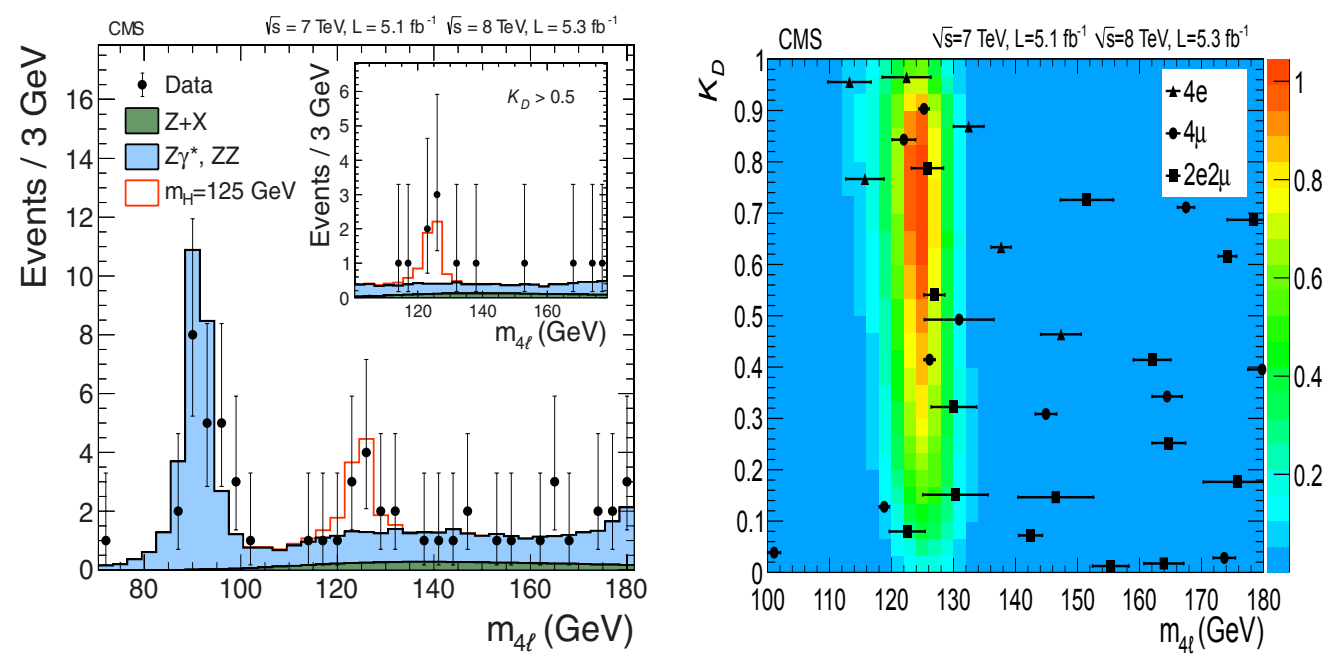

Figure 1: Distribution of the four-lepton reconstructed mass for the sum of the $4 e, 4 \mu$, and $2 e 2 \mu$ channels (left). Points represent the data, shaded histograms represent the background and unshaded histogram the signal expectations. Distribution of the MELA KD versus the four-lepton reconstructed mass $m_{4 \ell}$ (right) with contours shown for expected relative density of signal events for hypothesis $m_{H}=126 \mathrm{GeV}$. The points show data with measured invariant mass uncertainties.

and the rates are normalised to the values obtained using a method based on data. The measured distribution is well described by the SM background expectation.

The measured $\left(m_{4 \ell}, \mathrm{KD}\right)$ distributions are compared with the expectation from SM background processes, and exclusion limits at 95\% CL on the ratio of the production cross section for the Higgs boson to the SM expectation are derived. For this, the $\left(m_{4 \ell}, \mathrm{KD}\right)$ distributions of the selected events are split into six categories based on three final states and two running periods ( 7 and $8 \mathrm{TeV}$ ). These events are examined for 183 hypothetical Higgs boson masses in a range between $110 \mathrm{GeVand}$ $600 \mathrm{GeV}$, where the mass steps are optimized to account for the expected width, $\Gamma_{H}$, and resolution for the measurement of $m_{H}$ [42]. For each mass hypothesis, we perform a simultaneous likelihood fit of the six two-dimensional $\left(m_{4 \ell}, \mathrm{KD}\right)$ distributions using the statistical approaches discussed in Ref. [42]. As a cross-check, we have also studied one-dimensional $m_{4 \ell}$ distributions and found consistent, but systematically higher expected limits. We adopt the modified frequentist construction $\mathrm{CL}_{\mathrm{s}}[43,44,42]$ as the primary method for reporting limits. As a complementary method to the frequentist paradigm, we use the Bayesian approach [45] and find consistent results. 

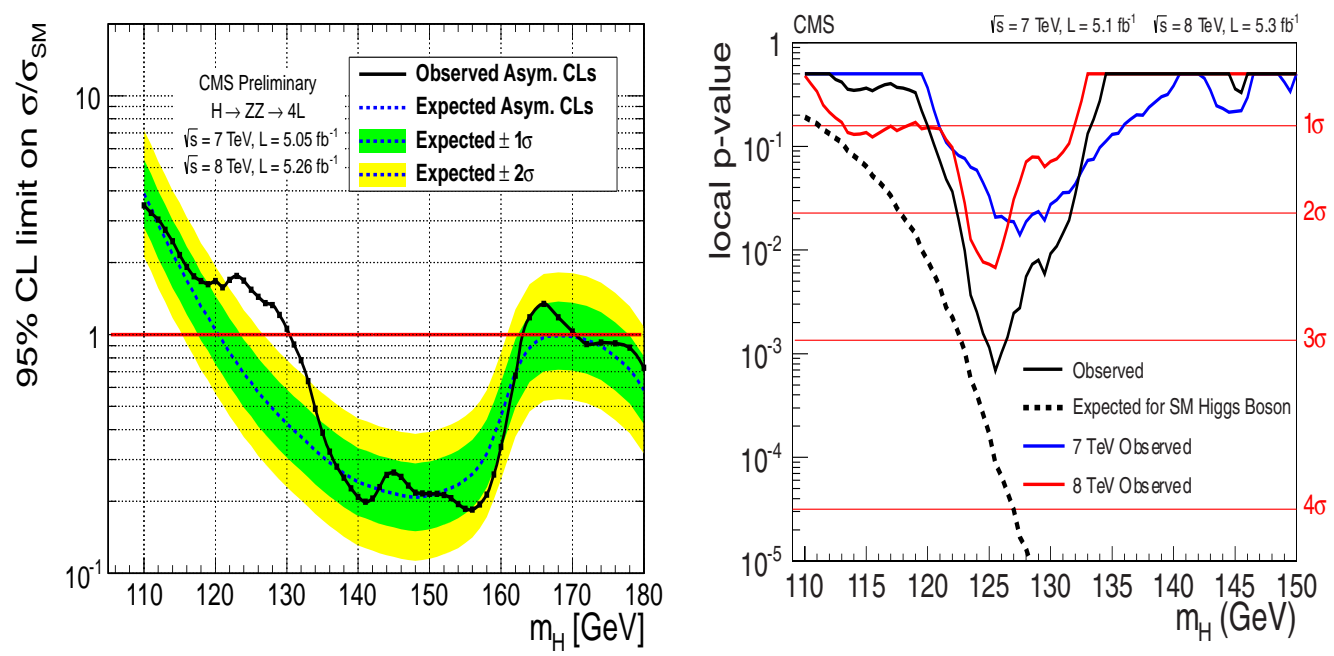

Figure 2: Observed and expected 95\% CL upper limit (left) on the ratio of the production cross section to the SM expectation. The $68 \%$ and $95 \%$ ranges of expectation for the background-only model are also shown with green and yellow bands, respectively. Significance of the local excess (right) with respect to the Standard Model background expectation as a function of the Higgs boson mass in the low mass range $110-180 \mathrm{GeV}$.

The upper limits obtained from the combination of the $4 \ell$ channels are shown in Fig. 2 (left). The SM Higgs boson is excluded by the four-lepton channels at 95\% CL in the range 131-525 GeV, except for the small range $162-172 \mathrm{GeV}$ where the branching ratio for the $H \rightarrow Z Z$ decay is disfavoured. The local $p$-values, representing the significance of local excesses relative to the background expectation, are shown for the low mass range as a function of $m_{H}$ in Fig. 2 (right). The minimum of the local $p$-value is reached around $m_{4 \ell}=125.5 \mathrm{GeV}$ and corresponds to a local significance of $3.2 \sigma$. The average expected significance for a Standard Model Higgs boson at this mass is $3.8 \sigma$ for the $2 \mathrm{D}$ fit.

To extract from the $4 \ell$ measurements a signal strength modifier $\mu$ for a SM Higgs boson signal $\left(\mu \times \sigma_{\mathrm{SM}}\right)$ and a most probable mass, we perform a likelihood scan on data in the 2D space of $m_{H}$ vs. $\mu$. At each point $\left(m_{H}, \mu\right)$, the likelihood is minimized with respect to all nuisance parameters. The global minimum is located at $m_{H}=125.6 \mathrm{GeV}$ and $\mu=0.7$.

\section{Conclusions}

The search covers Higgs boson mass hypotheses in the range $110<m_{H}<600 \mathrm{GeV}$. The analysis uses pp collision data recorded by the CMS detector at the LHC, corresponding to integrated luminosities of $5.05 \mathrm{fb}^{-1}$ at $\sqrt{s}=7 \mathrm{TeV}$ and $5.26 \mathrm{fb}^{-1}$ at $\sqrt{s}=8 \mathrm{TeV}$. Upper limits at $95 \%$ confidence level exclude the standard model Higgs boson in the range 131-162 and 172-525 GeV, while the expected exclusion range is $121-570 \mathrm{GeV}$. An excess of events is observed in the low $m_{4 \ell}$ mass range, making the observed limits weaker than expected in the absence of a signal. These events cluster around a mass $m_{4 \ell} \simeq 125.5 \mathrm{GeV}$, giving rise to a local excess with respect to the background expectation, with a significance of $3.2 \sigma$. This result constitutes evidence for a new massive state. 


\section{References}

[1] S. L. Glashow. Partial Symmetries of Weak Interactions. Nucl. Phys., 22:579, 1961.

[2] Steven Weinberg. A Model of Leptons. Phys. Rev. Lett., 19:1264, 1967.

[3] Abdus Salam. Weak and electromagnetic interactions. In Nils Svartholm, editor, Elementary particle physics: relativistic groups and analyticity, page 367. Almquvist \& Wiskell, 1968. Proceedings of the eighth Nobel symposium.

[4] F. Englert and R. Brout. Broken Symmetry and the Mass of Gauge Vector Mesons. Phys. Rev. Lett., $13: 321,1964$.

[5] Peter W. Higgs. Broken symmetries, massless particles and gauge fields. Phys. Lett., 12:132, 1964.

[6] Peter W. Higgs. Broken Symmetries and the Masses of Gauge Bosons. Phys. Rev. Lett., 13:508, 1964.

[7] G. S. Guralnik, C. R. Hagen, and T. W. B. Kibble. Global Conservation Laws and Massless Particles. Phys. Rev. Lett., 13:585, 1964.

[8] Peter W. Higgs. Spontaneous Symmetry Breakdown without Massless Bosons. Phys. Rev., 145:1156, 1966.

[9] T. W. B. Kibble. Symmetry breaking in non-Abelian gauge theories. Phys. Rev., 155:1554, 1967.

[10] Lyndon Evans and Philip Bryant. LHC Machine. JINST, 3:S08001, 2008.

[11] Georges Aad et al. Search for the Standard Model Higgs boson in the decay channel $H \rightarrow Z Z^{(*)} \rightarrow 4 l$ with $4.8 \mathrm{fb}^{-1}$ of pp collision data at $\sqrt{s}=7 \mathrm{TeV}$ with ATLAS. Phys. Lett., B710:383-402, 2012.

[12] Serguei Chatrchyan et al. Search for the standard model Higgs boson in the decay channel H to ZZ to 4 leptons in pp collisions at $\sqrt{s}=7 \mathrm{TeV} .2012$.

[13] Serguei Chatrchyan et al. Combined results of searches for the standard model Higgs boson in pp collisions at $\sqrt{s}=7$ TeV. Phys. Lett., B710:26-48, 2012.

[14] Georges Aad et al. Combined search for the Standard Model Higgs boson using up to $4.9 \mathrm{fb}^{-1} \mathrm{of} \mathrm{pp}$ collision data at $\sqrt{s}=7 \mathrm{TeV}$ with the ATLAS detector at the LHC. Phys. Lett., B710:49-66, 2012.

[15] ATLAS Collaboration. Combined search for the Standard Model Higgs boson in pp collisions at sqrt(s) $=7 \mathrm{TeV}$ with the ATLAS detector. 2012. Submitted to Phys. Rev. D.

[16] ALEPH, DELPHI, L3, OPAL Collaborations, and the LEP Working Group for Higgs boson searches. Search for the standard model Higgs boson at LEP. Phys. Lett. B, 565:61, 2003.

[17] CDF and D0 Collaborations. Combination of Tevatron Searches for the Standard Model Higgs Boson in the $W^{+} W^{-}$Decay Mode. Phys. Rev. Lett., 104:061802, 2010.

[18] ALEPH, CDF, D0, DELPHI, L3, OPAL, SLD Collaborations, the LEP Electroweak Working Group, the Tevatron Electroweak Working Group, and the SLD Electroweak and Heavy Flavour Groups. Precision Electroweak Measurements and Constraints on the Standard Model. Technical report, 2010.

[19] ALEPH, CDF, D0, DELPHI, L3, OPAL, SLD Collaborations, the LEP Electroweak Working Group, the Tevatron Electroweak Working Group, and the SLD Electroweak and Heavy Flavour Groups. Precision electroweak measurements on the $Z$ resonance. Phys. Rept., 427:257, 2006.

[20] S. Chatrchyan et al. The CMS experiment at the CERN LHC. JINST, 3:S08004, 2008.

[21] CMS Collaboration. Particle-flow event reconstruction in cms and performance for jets, taus, and met. CMS Physics Analysis Summary MS-PAS-PFT-09-001, 2009. 
[22] CMS Collaboration. Commissioning of the particle-flow reconstruction in minimum-bias and jet events from $p p$ collisions at 7 tev. CMS Physics Analysis Summary CMS-PAS-PFT-10-002, 2010.

[23] S. Baffioni et al. Electron reconstruction in CMS. Eur. Phys. J. C, 49:1099, 2007.

[24] CMS Collaboration. Electron reconstruction and identification at $\sqrt{s}=7$ tev. CMS Physics Analysis Summary CMS-PAS-EGM-10-004, 2010.

[25] CMS Collaboration. Commissioning of the particle-flow event reconstruction with leptons from $\mathrm{j} / \psi$ and w decays at 7 tev. CMS Physics Analysis Summary CMS-PAS-PFT-10-003, 2010.

[26] Matteo Cacciari and Gavin P. Salam. Pileup subtraction using jet areas. Phys. Lett., B659:119-126, 2008.

[27] S. Chatrchyan et al. Measurement of the Inclusive W and Z Production Cross Sections in pp Collisions at $\sqrt{s}=7 \mathrm{TeV}$. JHEP, 10:132, 2011.

[28] CMS Collaboration. Absolute calibration of the CMS luminosity measurement: Summer 2011 update. CMS Physics Analysis Summary CMS-PAS-EWK-11-001, 2011.

[29] LHC Higgs Cross Section Working Group. Handbook of LHC Higgs Cross Sections: 1. Inclusive Observables. CERN Report CERN-2011-002, 2011.

[30] A. Soni and R.M. Xu. Probing CP violation via Higgs decays to four leptons. Phys.Rev., D48:5259-5263, 1993.

[31] Vernon D. Barger, King-man Cheung, A. Djouadi, Bernd A. Kniehl, and P.M. Zerwas. Higgs bosons: Intermediate mass range at e+ e- colliders. Phys.Rev., D49:79-90, 1994.

[32] S Y. Choi, D J. Miller, M M. Muhlleitner, and P M. Zerwas. Identifying the Higgs spin and parity in decays to Z pairs. Phys.Lett., B553:61-71, 2003.

[33] B.C. Allanach, K. Odagiri, M.J. Palmer, Michael Andrew Parker, A. Sabetfakhri, et al. Exploring small extra dimensions at the large hadron collider. JHEP, 0212:039, 2002.

[34] C P. Buszello, I. Fleck, P. Marquard, and J.J. van der Bij. Prospective analysis of spin- and CP-sensitive variables in $H \rightarrow Z Z \rightarrow l(1)^{+} l(1)^{-} l(2)^{+} l(2)^{-}$at the LHC. Eur.Phys.J., C32:209-219, 2004.

[35] R M. Godbole, D J. Miller, and M. Margarete Muhlleitner. Aspects of CP violation in the H ZZ coupling at the LHC. JHEP, 0712:031, 2007.

[36] Wai-Yee Keung, Ian Low, and Jing Shu. Landau-Yang Theorem and Decays of a Z' Boson into Two Z Bosons. Phys.Rev.Lett., 101:091802, 2008.

[37] Oleg Antipin and Amarjit Soni. Towards establishing the spin of warped gravitons. JHEP, 0810:018, 2008.

[38] Kaoru Hagiwara, Qiang Li, and Kentarou Mawatari. Jet angular correlation in vector-boson fusion processes at hadron colliders. JHEP, 0907:101, 2009.

[39] Yanyan Gao et al. Spin determination of single-produced resonances at hadron colliders. Phys. Rev. D, 81:075022, 2010.

[40] A. De Rujula, Joseph Lykken, Maurizio Pierini, Christopher Rogan, and Maria Spiropulu. Higgs look-alikes at the LHC. Phys.Rev., D82:013003, 2010.

[41] James S. Gainer, Kunal Kumar, Ian Low, and Roberto Vega-Morales. Improving the sensitivity of Higgs boson searches in the golden channel. JHEP, 1111:027, 2011. 
[42] ATLAS and CMS Collaborations, LHC Higgs Combination Group. Procedure for the lhe higgs boson search combination in summer 2011. ATL-PHYS-PUB/CMS NOTE 2011-11, 2011/005, 2011.

[43] Thomas Junk. Confidence level computation for combining searches with small statistics. Nucl. Instrum. Meth. A, 434:435, 1999.

[44] A.L. Read. Modified frequentist analysis of search results (the $C L_{s}$ method). Technical Report CERN-OPEN-2000-005, CERN, 2000.

[45] Particle Data Group. Review of particle physics. J. Phys., G37:075021, 2010. 